\title{
Calling Time on Digital Clocks
}

\author{
David Sloan $^{1 *}$ \\ 1 DAMTP, Center for Mathematical Sciences, \\ Cambridge University, Cambridge CB3 OWA, UK
}

I explore two logical possibilities for the discretization of time, termed "instantaneous" and "smeared". These are found by discretizing a continuous theory, and the resulting structure of configuration space and velocities are described. It is shown that results known in numerical methods for integration of dynamical systems preclude the existence of a system with fixed discrete time step which conserves fundamental charges universally, and a method of avoidance of this "no-go" theorem is constructed. Finally the implications of discrete time upon quantum cosmology are discussed.

NB : At this point this paper is a draft, citations and formatting are not completed.

*Electronic address: djs228@hermes.cam.ac.uk 


\section{INTRODUCTION}

"The now is a link of time....for it links together past and future, since it is a beginning of one and an end of another." - Aristotle [1]

Whether physics can be described on a continuum or lattice is one of the oldest questions considered by philosophers in one form or another. The most famous paradox of Zeno argues against the infinite divisibility of a temporal interval - that is against continuous time[2]. Achilles is sent to chase a tortoise, which is given a head start. If we label the position of the turtle $x_{t}$ for points in time $t$. Then in each instant that Achilles reaches $x_{t}$ the turtle has moved on to $x_{t+1}$, thus it should seem logically impossible for Achilles to catch the turtle. Viewed externally, however, one can easily verify that Achilles does indeed catch the turtle at a finite time. Of course we now know the resolution to this apparent paradox is that there can be a finite sum of an infinite number of terms, as Archimedes found. To Zeno, however, this was not known, as it was assumed that an infinite sum cannot be finite, and thus it appeared that there could not be an infinite number of time points in an interval time should not be continuous.

Tong makes a case against the a lattice reality based upon the problems with implementation in practical terms [3], stating " no one knows how to formulate a discrete version of the laws of physics." Futhermore he makes the compelling case that chiral fermions do not sit easily upon a lattice, and since the Standard Model is a chiral model, this means that it appears impossible to place known physics upon a lattice. Indeed lattice simulation models of chiral fermions in four dimensions seem to rely crucially upon treating the particles as living essentially on a five-dimensional lattice [4]. There is an important distinction to be noted here: Attempts are made to simulate the four-dimensional behaviour of the particles, for which the use of extraneous mathematical structure (in this case the extra dimension) is appropriate. If, however, one were to claim that physics in fact inhabits a lattice, rather than being simulated on one, this extra structure becomes unwelcome baggage whose existence must be explained. Tong goes on to argue that the appearance of the integers in physics is constructed from an underlying continuum, an argument which mirrors the duality between a particle which exists as the excitation of a field, and a field which is observed to be composed of particles.

There are three ways in which one can respond to arguments of this type. The first, more simplistic argument is to state that what has been shown is not a "no-go" theorem against lattice constructions in four dimensions, merely that we do not yet know how to construct one. As such it is plausible that a lattice construction may be achieved in the near future, at which point all such objections would be rendered null. A second point would be that the problem may come about from trying to force a continuum theory onto a lattice. If discreteness is fundamental, then a continuum theory should emerge at large scales, but features of theory at the lattice level may be radically different from those of the continuum. The final method of avoiding such problems in the context of this paper is to argue merely in favour of discrete time, not space. This argument may seem unnatural on some level, as the even-handed treatment of time and space is a guiding principle for modern theories such as relativity. However it is clear that there is a physical, substantive distinction between the two at least at the level of metric signature. In practical implementations of these theories a space-time splitting is employed regularly.

In this paper I will explore the effects of introducing a discrete tick to physical systems. 
The paper is laid out as follows: In section III I will show how the effects of introducing this tick onto systems with continuous time parameter, establishing the kinematics of such treatments. This is followed by section IV in which I discuss the implementation of discrete time steps in numerical simulations. Section V shows one practical application of these techniques to circular motion, and a way of establishing dynamics which solve some the problems found is introduced in section VI. Finally I will note how this effects quantum gravity. But first, to clocks...

\section{A NOTE ON CLOCKS}

"They took away time, and they gave us the clock." - Abdullah Ibrahim.

The nature of physical clocks seems dichotomous at first glance. A clock is a timekeeping device, an instrument whose observation gives information used to define the interval between two events. A clock should contain a cyclic element, which describes the tick of the system. This role is performed by, for example, observations of the positions of shadows cast by the sun or the repeated dripping of water from a vessel (as was used in the earliest clocks of Egypt and Babylon) through to the oscillations of a caesium atom used in the atomic clocks of today. The clock must also be monotonic, defining unambiguously a separation of reality into past and future.

There is of course no contradiction in this. Although at first an individual system cannot be seen to be both globally monotonic and cyclic, a clock is not, in essence, a single system. Clocks consist of two distinct coupled systems, these being the cyclic and monotonic parts accordingly. The cyclic part triggers, at some point in its cycle, a distinct and discrete advance of the monotonic part, as the pendulum of a grandfather clock causes the second hand to tick upon reaching its escapement, advancing the second hand. Of course, a grandfather clock is cyclic in itself, but upon marking the end of each day, a calendar can be updated such that the overall observation of time remains monotonic.

As described thus far, the measurement of time may be refined by reducing the interval of a tick and classically there is no reason that this refinement may not, in principle, yield an arbitrary degree of accuracy. However, lurking in the small scales is the spectre of quantum mechanics and the Mandelstamm-Tamm uncertainty which effectively means that for any quantum clock there is an unavoidable minimum for the amount of time it takes for a wavepacket to move a distance equal to its standard deviation, for example. For a comprehensive review see [13]. This minimum is dependent upon the physical nature of the clock, so one might suspect that it is merely a practical problem to refine the tick indefinitely. However, it is conceivable that time is fundamentally discrete, with an indivisble tick.

A prime candidate for discretization is the Planck time - the unique time that can be formed from the dimensional constants of nature (Newton's constant, Planck's constant and the speed of light). The Planck time, around $10^{-42}$ seconds, is the time interval after the big bang on which quantum gravity effects are thought to be dominant, and the time-scale on which we would expect to see quantum corrections to Einstein's equations. The upshot is that the dynamics of the tick may in fact be unavailable as an observable, and thus the only reading of time one can get is that of the monotonic part, reading time as though from a digital clock.

Within Quantum Gravity, issues regarding implementation within the Hamiltonian framework are so severe that they have been dubbed "The Problem(s) of Time". Some state that 
this consists of as many as eight separate yet connected issues [14]. The purpose of this paper is not to address such issues, but I will point out that even in the symmetry reduced mini-superspace models which are used ubiquitously in quantum cosmology time evolution is measured with respect to a scalar field. If one is even-handed in treating both geometrical and matter variables, one must apply the same "polymer quantization" [6] to both, and thus the universe is imbued with a discrete tick.

The role of a clock within a physical system is split into three parts by Busch $[11,12]$. First, time as measured may be "external" or "pragmatic" - there is no coupling between the dynamical system being observed and the clock used to measure time within that system. In a classical sense, external time can be said to be measuring some aspect of Newton's absolute time on which dynamics takes place. Second, an "intrinsic" time is one which is measured as some quantity of the system itself, such as the readout of a digital display, or the position of the hands on the face of a watch. Third, "observable" or "event" time is a direct measurement of some physical quantity which is taken to be time itself, such as the position of a particle. Throughout this paper I shall always have the idea of intrinsic time in mind, as an external time can be made intrinsic simply by extending the configuration space of a system with external time by taking the product of a the configuration space with the configuration space of the clock. To those interested in relational observables, such as the cosmologist, intrinsic time is all one can work with - there is no external space on which a clock can live. In terms of quantum gravity, any physical clock must have a mass and thus interact gravitationally with all other components of the system through its action on space-time. Therefore cosmologically all observable time is intrinsic.

\section{DISCRETE TIME}

"God made the integers, all the rest is the work of man." - Kronecker.

In this section we will discuss the result of overlaying a discrete temporal structure onto a continuum dynamical system. The result of this will be to produce a system which contains only information about the discrete structure, removing all reference to the continuum. The background continuum is therefore used in the manner of Wittgenstein's ladder [7] an external structure of convenience whose utility has no bearing on the resultant system. Constructions of this type are commonplace in physics. Prime examples include the use of a fiducial cell in cosmology against which to measure the size of the universe. Upon calculation, care must be taken to show that results are independent of the choice of cell. Similarly, General Relativity is a background independent theory, yet in practice calculations often involve coordinate choices. Again resulting physical quantities must be shown to be coordinate independent.

The mathematical structure of our background system shall consist of a manifold $\mathcal{M}$ of arbitrary dimension $n$ which can be factlorized into configuration ${ }^{1}$ states and a onedimensional $^{2}$ temporal direction : $\mathcal{M}=\mathcal{C} \times \mathcal{T}$. The global topology of $\mathcal{M}$ will remain un-

\footnotetext{
${ }^{1}$ Here we shall work extensively in the position representation, but extension to the momentum representation of states is straightforward.

${ }^{2}$ It is conceivable that this is extended to multiple temporal directions, however this action seems to offer little to the discussion at hand other than to distract from the essential argument through mathematical
} 
determined at present, however our underlying continuum mean that both $\mathcal{C}$ and $\mathcal{T}$ should be continuous. For reasons of convenience we will often think of $\mathcal{T}$ as being either $S^{1}$ (the circle) or $\mathbf{R}$ (the real line). Here we follow the construction of the " $3+1$ decomposition" of Arnowitt, Deser and Misner [8], however it is important to note that we are not splitting space-time but rather configuration-space-time. Physically time here is a further configuration variable, that of the system clock.

\section{A. Treatment of position}

The discretization of time can be performed in two distinct ways, which can be thought of as describing configurations instantaneously or over an interval. To illustrate this, let us consider two physical scenarios which each will describe a discretization of time on a particle moving in a circle. In the first situation the system is measured at fixed intervals with a stroboscopic light, whose flash is interpreted as being instantaneous ${ }^{3}$. This I will call "instantaneous measurement".

A second method would be to consider measurement to be smeared across an interval. This is effectively the complement of the above: a camera is set up to measure the system with its aperture open for an interval, closing instantaneously between intervals. The resulting measurement will not consist of a unique configuration but rather a trajectory across the interval which is to be considered a single measurement. Here the idea of an instant is used in analogy to that of Le Poidevin [16], in which an instant separates two intervals. Le Poidevin took this instant to be the "now" which separates past from future, I shall invoke it to separate temporal intervals. This treatment I will term "smeared measurement".

Both measurements of a system's position consist of replacing the time interval of our manifold by a discrete subgroup, for instance replacing a real interval $\mathcal{I}$ with a subset of the integers $\mathbf{Z}$ or replacing $S^{1}$ with $S_{n}$ (the cyclic group of order $n$ ). Without loss of generality let us take each tick of the clock to be separated by an equal time interval with respect to the background time. This can be achieved by making a transformation of the background time, since an operational time obtained through any monotonic function of background time will serve will equal sufficiency. In replacing a continuous set with a discrete one we throw away an infinite amount of information about the system - the complete dynamics between "ticks of our clock.

To give an illustration of the differences between our two treatments consider the motion of a particle along a path. Suppose we describe the motion with background dynamics $x=x(t)$. One may ask for the relational observable $X_{T}$ of a system, defined to mean the position of a particle at the time when the clock reads time to be $T$. In the instantaneous treatment $X_{T}$ will be a single reading of a number, the unique position at that instant in background time. However in the smeared treatment the measurement will be a complete path, all positions occupied between background times $T$ and $T+\delta T$ for temporal intervals $\delta t$.

"Instants are not parts of time, for time is not made up of instants any more than a magnitude is made of points" [9]

Some take instantaneous treatment of time is taken to induce a discretization of position

complexity.

${ }^{3}$ This could be the result of measuring a single photon reflected from a particle 
space. Zeno's Dichotomy paradox [5] argues that one must occupy each point in a spatial interval whilst traversing the interval. In doing so, if time is discrete, one must take an infinite number of time steps, one at each position. This argument relies (unknowingly at the time of its inception) on the cardinality of any interval $\mathcal{I} \subset \mathcal{R}$ being larger than that of $\mathcal{Z}$ - there can be no mapping $f: \mathcal{Z} \rightarrow \mathcal{I}$ such that $f$ is surjective. However in our treatment the motion is not taken to be a continuous one in discrete time - from one tick to the next one's position can change by an amount that is not infinitesimal.

The smeared treatment affects position space on a more fundamental level. Observations of position are no longer points, but rather (unordered) paths in the configuration manifold. In performing the smearing all information about the velocity through position space during a tick is lost - all that remains is a trajectory consisting of all the points occupied during the interval. As an example, consider a particle moving in one dimension, its position here during a tick would be the entire interval covered between its leftmost and rightmost positions covered by the continuum trajectory in this interval. Our observations will consist of a set of paths $\gamma_{t}:[0,1] \rightarrow \mathcal{C}$ modulo an equivalence relation identifying two paths formed by any permutation of the unit interval. For continuous dynamics, this permutation should be smooth, but in principle there is no distinction for sudden leaps. Note that we have lost all sense of direction of the path - we cannot say from a single path whether the a particle moves left to right, or vice versa, or even starts and ends somewhere in the middle of the path, reaching ends at intermediate times. A path in which a particle revisits a previous point during a tick is permissable, and cannot be distinguished from one which does not.

If our background system is does not revisit the same point in position space during a two consecutive ticks there will be a unique point at which the paths are joined, and this may be taken to be the configuration of the background system at the instant between ticks. However if at any stage in these ticks a point in configuration space is revisited this reconstruction is no longer possible: As an example, consider a particle moving in a single dimension of which we make two observations. During the first tick we observe the path to cover the interval from 0 to 2. During the second, from 1 to 2 . We cannot say, from these two observations whether the background dynamics had the paths join at 2, 1 or in fact any position in between, as all such dynamics would yield the same observations.

\section{B. Treatment of velocities}

Once position space has been defined, it is necessary to define velocities which will be fundamental to understanding dynamics. The usual method of defining a velocity is not available since defining

$$
\dot{x}=\operatorname{Lim}_{\delta t \rightarrow 0} \frac{x(t+\delta t)-x(t)}{\delta t}
$$

relies crucially on this limit being accessible to the system. In a discrete time system, $\delta t$ has a fundamental minimum, and furthermore, in the case of the smeared treatment, the action of subtraction of two intervals is not obviously defined. Let us first address the simpler case of instantaneous time.

The fletcher's paradox outlines a key difficulty in identifying velocity in instantaneous time. It can be formulated in the following way: Suppose one is given a set of instantaneous observations of a particle in motion (Zeno uses an arrow in flight). Then at each instant the particle has a fixed position and does not appear to move, and so there appears to be 
no motion in sum. This is resolved by Russell's "at-at" treatment of motion [10] - that to travel between two points at different times is to occupy all intermediate points in the interval between.

In modern differential geometrical terminology, we describe the velocity of an object as a member of the tangent space to the position space at the point occupied by the system: $v \in \mathcal{T}_{x} \mathcal{C}$. One can define the obvious velocity in this instance by simply letting the removing the limit in 3.1. However, velocities defined this way are not necessarily members of $\mathcal{T}_{x} \mathcal{C}$. Recall that $\mathcal{T}_{x} \mathcal{C}$ is isomorphic to $\mathbf{R}^{n}$, where $n$ is the dimension of the manifold $\mathcal{C}$. For a finite tick, $v \delta t$ must be a automorphism of mathcalC. In fact, the space of velocities at a point on $\mathcal{C}$, which we shall denote $\mathcal{V}_{x} \mathcal{C}$ must be isomorphic to $\mathcal{C}$, since velocity at a point is defined by the point in $\mathcal{C}$ at which one arrives after a single tick. Upon approach to the limit, the two points in the configuration space become close and once again we recover the tangent space. This naturally carries over to momentum which is no longer a member of the co-tangent space (the space of linear functionals of members of the tangent space) but in fact lives on the co-velocity space - the space of linear functionals of members of the velocity space.

Velocity in the smeared treatment is more complicated. Our observations consist of unordered paths in configuration space. In the case where any two adjacent paths overlap only in a unique point, one could define the velocity of a particle to be the length of the path covered divided by the length of the tick. However this gives only a minimum for the velocity of the particle in the background system, as the particle may not have uniformly covered this interval, but may have oscillated multiple times along the path during the tick. Consider the case of a man pacing back and forth on a boat - in a smeared observation he will appear to move with the approximate velocity of the boat, but on a closer inspection his speed at any time will not be this. Thus we obtain a lower bound for speed, defined to be the length of the path covered at any interval. This lower bound is the only reasonable candidate for a velocity in this setting, though we must assign a sense of direction to it. This is simple to do in the case that intersections are unique, but for multiply overlapping intervals the task becomes difficult. One possible assignment is to take the velocity as being oriented towards any part of a later path that is not contained in the prior one, or towards any point not contained in the subsequent path, but this of course does not deal with paths contained entirely within the both the preceding and subsequent path. In these cases the only seemingly logical thing to do is to set the velocity to zero.

Thus our space of velocities becomes the space of directed paths in $\mathcal{C}$. If $\mathcal{C}$ is compact, or indeed any configuration variable on $\mathcal{C}$ is compact, the space of velocities is again removed from $\mathcal{T}_{x} \mathcal{C}$. Those positions inhabiting a compact direction of position space must induce a finite dimension in velocity space.

Implementation of dynamics in the smeared system is considerably more difficult: The logical method would be to apply a similar form of numerical integration to the entire path, taking each point and moving it by the velocity of the path. In the case of a particle moving along a line, this is of course perfectly acceptable. However there is a question of accelerations, which are brought about by the application of force.

\section{DISCRETE TIME AS NUMERICAL INTEGRATION}

"'It always bothers me that, according to the laws as we understand them today, it takes a computing machine an infinite number of logical operations to figure out what goes on in 
no matter how tiny a region of space, and no matter how tiny a region of time. How can all that be going on in that tiny space?" - Feynman.

The difference between velocity space and tangent space is known to those working on numerical integrators, though in different terms. These methods attempt to reproduce the continuum dynamics with a finite (though often variable) time-step. The Ströminger-Verlet method [17] (and improved velocity-Verlet [18]) uses the velocity and acceleration of the system both at an instant and in the preceding and succeeding instants to refine estimates of discrete motion to remain close to the continuum trajectory. The Runge-Kutta algorithm [19] is a more complex method involving the use of iterative methods to perform a timestep. One might be tempted, therefore, to state that a discrete system should run on a very accurate numerical integration method.

However, there is a problem with this system: The dynamics of the system of $t_{n}$ depend upon an estimation of the configuration and hence accelerations of the system at $t_{n+1}$. Unless we allow for the (potential) future to act upon the present, this is not possible: A particle cannot know the accelerations it would feel if it were to move to a future location until it moves there. If one insists that all the information available to a particle is its current position and velocity (or momentum), none of these methods can be applied. Even if one were to allow it access to its complete prior history over discrete time, this would be insufficient, for one can construct two paths (using Hermite polynomials for a one dimensional example) each of which visit all prior configurations but differ on future locations. One is then driven towards describing the dynamics of discrete time using a method more akin to that of Euler for the numerical integration of a differential equation. This is clearly not optimal, as it will break conservation equations held in the background continuum (an example of which is given below). It has been shown by Ge and Marsden [15] that there is no fixed time step method which conserves symplectic structure, and Noether charges (ie all conserved quantities in the continuum system). In fact, all known numerical integrators which do conserve Noether charges require knowledge of the forces that the system will experience after the time-step has been implemented. In simulations this is perfectly acceptable, as one can tell the computer what model of physics to implement. However in reality this would require information about the future to be passed to a prior time, which is for most an unpalatable premise.

Thus one is forced into one of the following: We could drop the notion of discrete time as it appears incompatible with conservation laws. This is of course the most obvious route to take, but as I will argue in section VII there is good reason to believe that a discrete tick does exist. The second possibility is that a the continuum implications of Noether's theorem cannot apply in a discrete time setting, so physics breaks all conservation laws at some level. Since symmetry and conservation lie at the very heart of all modern physics, this is a very unwelcome path to take. Finally one could argue that dynamics must be altered in some fundamental way when one discretizes time. This I discuss further in section VI.

\section{CYCLIC CONFIGURATIONS}

The limitations of discrete treatments of time become apparent when one treats cyclic systems whose period with respect to background time is of the same order as the length of a tick. In such cases our ideas of basic physical quantities such as velocity can become ill-defined. We shall illustrate this problem by exploring a simple system: uniform circular motion. This systems has a long-standing association with the idea of time as for centuries 
time has been displayed on a clock through the uniform circular motion of its hands. It is therefore fitting that this forms an exemplary model of issues in the discretization of time.

\section{A. Uniform Circular Motion}

Let us consider a simple system, the motion of a particle travelling with uniform angular velocity around a circle. The dynamics of our system is simple to describe: $\theta(t)=\theta_{0}+\omega t$, in which $\omega$ is the angular velocity. For brevity of exposition let us choose a gauge in which $\theta_{0}=0$. Let us define the period of the system $\tau=2 \pi / \omega$.

In the instantaneous treatment of this system an observer makes a number measurements $\theta(t)=0, \theta_{1} \ldots \theta_{n}$ corresponding to the position of the particle at clock times 1 through $n$. However, upon being asked to reproduce the angular velocity of the system, such an observer runs into immediate ambiguities: She cannot determine whether or not the particle is moving clockwise with angular velocity $\theta_{1} / \delta t$ or anticlockwise with angular velocity $\left(2 \pi-\theta_{1}\right) / \delta t$. In fact, to the observer all background dynamics of the form $\omega_{n}=\omega+2 \pi n / \delta t$ for $n \in \mathbf{Z}$ will yield indistinguishable observations. If $\delta t$ is a multiple of $\tau$ the observer will not see the particle move at all. This is well known through the stroboscopic analogy as an optical illusion which has in the past been used to determine the frequency of, for example, cylinder firing in car engines.

This is a clear illustration of the difference between $\mathcal{V}_{x} \mathcal{C}$ and $\mathcal{T}_{x} \mathcal{C}$. The tangent space to the circle is the real line, but the topology of the space of velocities distinguishable with instantaneous time is that of the circle itself.

Let us consider dynamics, supposing that the circular motion of our particle is brought about by some central force. If one were to adopt a strict implementation of discrete time, taking as a basis for dynamics Newton's laws of motion, and updating both velocity and acceleration only once per time step (ie implementing the method of Euler) there is an obvious problem. Newton's laws of motion state that we must evolve motion along the tangent to the curve described (the velocity should be a member of $\mathcal{T}_{x} \mathcal{C}$. This immediately leads to the particle leaving the circle, breaking energy and angular momentum conservation. One could ask how bad this problem is: To first order in each of the variables involved the drift of the numerical integration increases as $\omega^{2} r T \delta t / 2$. Where $T$ is the total time interval, divided into ticks $\delta t$, for a particle orbiting at radius $r$. For the orbit of the Earth around the sun since its formation this distance comes to around $2 \times 10^{-17} \mathrm{~m}$ if we assume the fundamental tick to be the Planck time. This is far less than the radius of a proton, for example, thus we are unlikely to be able to detect any effect of this granularity, even taking fast spinning objects such as pulsars into consideration.

One might argue that the implementation should involve some averaging or weighted average of an initial velocity and a final velocity, $v_{f}=v_{i}+a \delta t$ and so the implemented velocity for the tick should be $v_{a}=\left(v_{i}+v_{f}\right) / 2$. However, this does not alleviate our problem - since it is simple to show that the final speed will be higher than the initial speed, thus the system has not conserved energy despite the presence only of a central force. One quickly runs into the theorem of Ge and Marsden[15] with such efforts.

At first sight the smeared treatment appears advantageous: It is indeed possible to determine the direction and angular velocity of the system for small values. For $\delta t$ smaller than $\tau$ the measurement of angular velocity can be performed by simply taking length of the complete interval covered in each tick and dividing by the length of the ticks. However, once the tick length becomes greater than or equal to the period of motion, all observations 
become indistinguishable, as each observation will yield the entire circle as the path covered in that tick. If the tick length is one half the period of motion or greater, we will be unable to distinguish in which direction the particle is moving without resorting to a trick: It will be moving counter to the direction in which the paths defined as the complete circle minus the path covered in a tick is moving. This again is ambiguous at exact division of the period into half.

\section{NEW DYNAMICS}

If one wishes to keep both discrete time and conserved quantities in the manner of continuum systems, then one is forced to implement dynamics in a way that differs from the usual methods of Newton, Lagrange et al. Classical evolution is implemented through the use of a second order differential equation:

$$
\ddot{q}=f(q, \dot{q})
$$

From this prescription one can find the trajectory of a system. However as has been noted above, if one implements finite time-steps the trajectory that one recovers differs from that of the continuum, since evolution through a finite length along a vector in the tangent space does not necessarily remain in the space compatible with conservation of Noether charges.

One way in which conservation can be implemented is to enforce "by hand" conservation which is brought about by symmetries of the system. I shall construct such a system to show that in principle such systems can exist, without making claims as to their accuracy or fidelity to known physics. This manner of ensuring that fundamental physical quantities such as energy and momentum are conserved is similar in spirit to that used in discrete Hamiltonian systems in engineering for example [20].

The method is implemented as follows: For a system, establish those charges which are to be conserved due to symmetry using the method of Noether. For an initial value problem, find the surface(s) within phase-space such that this conservation takes place, $\mathcal{E}$.

Dynamics is implemented by using finite time-step methods with the fundamental tick: $q^{\prime}(t+\delta t)=q(t)+\dot{q}(t) \delta t, \dot{q}^{\prime}(t+\delta t)=\dot{q}(t)+f(q, \dot{q}) \delta t$. Finally one finds the point of $\mathcal{E}$ such that $q, \dot{q}$ are closest (with respect to some given metric) to $q^{\prime}, \dot{q}^{\prime}$.

Implementing this method in the case of an inverse square central force yields approximately the correct dynamics: Conservation of the angular momentum and energy are implemented, and if one measures distances in configuration by $D:=\sqrt{\tau^{2} \Delta \dot{q}^{2}+\Delta q^{2}}$ then one finds that the orbit recovers the clasical limit when $\dot{r} \tau$ is small compared to $r$.

This method has clear limitations. It depends upon the choice of measure used on configuration space to define a notion of distance from the conservation surface. Further the link between velocity and position become somewhat tenuous - velocity is no longer measurable directly as a change in position, but rather is an intrinsic quality of the system. Of course, this is the nature of any instantaneous velocity measured in a discrete system as we have seen, but nonetheless this remains aesthetically displeasing. The dynamics expressed here will differ considerably from continuum dynamics in the case of chaotic systems, as surfaces of configuration space compatible with conservation of fundamental charges may become close to one another, allowing a system to jump from one classical trajectory to the next.

Despite the limitations, what we have established is a method by which a discretized system may retain conservation laws mirroring those of the classical system on which it 
is based. The method is contrived and arbitrary, relying heavily on implementation of conservation by fiat, but is proof that such solutions can exist without having to resort to retrodiction of motion in the manner used by numerical integrators, or varying of time step.

\section{DISCRETE TIME AND QUANTUM GRAVITY}

There have been several attempts to quantize gravity through a discretization of geometry. Perhaps the most famous of these is Regge calculus [21] in which space-time is broken down into blocks which are internally flat (ie the inside is a section of Minkowski space) and whose curvature lies entirely at the interface between two blocks. This method has the appearance of breaking up a curve into a series of straight lines, with all the curvature being concentrated at the corners.

The causal dynamical triangulations programme [22] makes a splitting of space-time into space and time. Time is implemented in discrete steps, with the layer between being "triangulated" by a set of polyhedra. Once again, curvature lives on the interface between these polyhedra. The programme attempts to reconstruct a path-integral approach to quantum gravity by restricting the integral over geometry to being one over such triangulations. This has all the hallmarks of an instantaneous discretization of time - each layer of polyhedra is overlaid on the last at a discrete time interval. Sorkin's causal set model [23] follows a similar scheme, but rather than triangulating space, takes a discrete "sprinkling" of points into space-time to create its structure. Time here would again appear instantaneous for any finite number of points, though the interval of any time step would be arbitrary.

Collins et al. [24] show that when one takes into consideration the interactions of elementary particles a very tight bound is placed upon the breaking of Lorentz invariance. A granularity of space-time on the Planck scale does not necessarily induce a factor of $E / E_{p l}$ in corrections to the continuum model for a system of energy $E$ (wherein $E_{p l}$ denotes the Planck energy). Does this rule out discrete time?

Rovelli and Speziale [25] argue that this is not in fact the case for discretization of length. Just as the discretization of angular momentum does not spoil rotational invariance, the existence of a minimal length (or in the case considered by Rovelli and Speziale area) does not break Lorentz invariance. A Lorentz transformation between two observers necessarily alters the operators used to measure length, which will not commute. It is entirely plausible that an analogous argument can be made for any measurement of time.

As I have previously noted, in treatments of cosmology, time must be intrinsic in the manner of Busch - there can be no external parameters which are used to describe time. Modern treatments of quantum cosmology, such as Loop Quantum Cosmology, employ a matter field as a clock. In the simplest models this is a massless scalar field, so its motion is monotonic, and observations of the field value give a time for the system [26].

In simplicial decompositions of space, such as those used in spin-foam theories, time will again appear to have a discrete nature. Here one decomposes four dimensional space into a set of simplices which fill a region, and a time slicing can be applied by cutting across these simplices at an instant. However, due to diffeomorphism symmetry, two slices are indistinguishable if the networks dual to the simplices have the same structure, so when viewed from this perspective, time is simply the evolution of networks, which are inherently discrete - only the topology of a network is important as there is no notion of geometry. Therefore each change to the network, such as adding a new link or node, is a discrete step, and must appear to happen instantaneously in the continuum theory, or in a "tick" in the 
discrete theory.

There are two obvious issues that must be addressed when applying discrete time to cosmology. The first is that the Planck time is precisely the time at which quantum effects are thought to be dominant. However, by our definition there would only be a single tick between this point and the singularity itself. Within LQC, there may be as few as 20 ticks during which the density is high enough for quantum effects to be significant. Yet these differences are to be the time in which quantum gravity replaces GR. It appears unlikely that such a narrow set of points can have so large an effect on global dynamics.

The second problem to be addressed is that if one does have singularity resolution in a continuum theory due to some modified dynamics in which the universe "turns around" from contraction to expansion, the turn around could be missed in the discrete time step. As an analogy, consider a particle moving in a one dimensional potential well towards a thin yet high wall. In the continuum theory, the particle will reflect off the wall and its trajectory reverse, but in the discrete theory a high enough velocity will allow the particle in some cases not to "see" the wall at all - it will simply be on one side at one time and the opposite one tick later. In LQC this scenario is avoided as the singularity is disallowed by the constraint, but in other theories in which singularity resolution is brought about, this effect may be significant.

\section{Acknowledgments}

I am very grateful to Brian Pitts and particularly Jeremy Butterfield for discussions and proof-reading an early draft of this work I acknowledge support from the Templeton foundation as part of the Establishing the Philosophy of Cosmology project.

\section{BIBLIOGRAPHY}

[1] Aristotle 1996, Book IV, 222a10

[2] Aristotle-Physics VI:9, 239b5

[3] Tong, David Physics and the Integers 2011

[4] Kaplan, David A Method for simulating chiral fermions on the lattice Physics Letters B 288 (3), 342-347

[5]

[6] Alejandro Corichi, Tatjana Vukainac4,, and Jos A. Zapata Polymer quantum mechanics and its continuum limit 2007

[7] Wittgenstein, L. Tractatus Logico-Philosophicus 1921

[8] Arnowitt, R.; Deser, S.; Misner, C. "Dynamical Structure and Definition of Energy in General Relativity". Phys Rev 1161959

[9] Aquinas. Commentary on Aristotle's Physics, Book 6.861

[10] Huggett, Nick (1999). Space From Zeno to Einstein.

[11] Busch, P. (1990), 'On the energy-time uncertainty relation: Part I: Dynamical time and time indeterminacy', Foundations of Physics 20 , pp. 1-32. 
[12] Busch, P. (1990a), 'On the energy-time uncertainty relation: Part II: Pragmatic time versus energy indeterminacy', Foundations of Physics 20, pp. 33-43.

[13] Butterfield, J. (2012), 'On time in quantum physics', forthcoming in The Blackwell Companion to Time, edited by A. Bardon and H. Dyke; Oxford: Blackwell; available at http://philsciarchive.pitt.edu/9287/

[14] Anderson, Edward The Problem of Time in Quantum Gravity, 2010

[15] Ge, Z. and J. E. Marsden [1988] Lie-Poisson integrators and Lie-Poisson Hamilton-Jacobi theory, Phys. Lett. A 133, 134139

[16] Le Poidevin, R. (1997). Time and the Static Image. Philosophy, 72, 17588.

[17] Kane, Marsen, Ortiz, Journal of Mathematical Physics 40, 1999, 33533371

[18] Simo and Gonzalez, Recent Results on the Numerical Integration of Infinite Dimensional Hamiltonian Systems

[19] Süli, Endre; Mayers, David (2003), An Introduction to Numerical Analysis, CUP

[20] O. Gonzalez, Time integration and discrete Hamiltonian systems, Journal of Nonlinear Science 6 (1996) 449467.

[21] T. Regge (1961). "General relativity without coordinates". Nuovo Cim. 19 (3): 558571

[22] R. Loll, Discrete Lorentzian Quantum Gravity, arXiv:hep-th/0011194v1

[23] D.P. Rideout, R.D. Sorkin; A classical sequential growth dynamics for causal sets, Phys. Rev D, 6, 024002 (2000) arXiv:gr-qc/9904062

[24] John Collins, Alejandro Perez, Daniel Sudarsky, Luis Urrutia, Hctor Vucetich Lorentz invariance and quantum gravity: an additional fine-tuning problem? 2004

[25] Carlo Rovelli, Simone Speziale Reconcile Planck-scale discreteness and the Lorentz-Fitzgerald contraction 2003

[26] Abhay Ashtekar, Tomasz Pawlowski, Parampreet Singh Quantum Nature of the Big Bang 2006 